\title{
The role of serum and urinary biomarkers in the diagnosis of early diabetic nephropathy in patients with type 2 diabetes
}

\author{
Deyuan Zhang ${ }^{1,2}$, Shandong Ye ${ }^{\text {Corresp., } 1,3}$, Tianrong Pan ${ }^{2}$ \\ ${ }^{1}$ School of Medicine, Shandong University, Jinan, Shandong, China \\ 2 Department of Endocrinology, The Second Affiliated Hospital of Anhui Medical University, Hefei, Anhui, China \\ 3 Department of Endocrinology, Anhui Provincial Hospital, Hefei, Anhui, China \\ Corresponding Author: Shandong Ye \\ Email address: yesd196406@163.com
}

Background: Previous studies have shown that a variety of biomarkers are closely related to the occurrence and development of early-stage diabetic nephropathy (DN) in patients. The aim of this study was to evaluate the role of multiple sera and urinary biomarkers in the diagnosis of early-stage DN in patients with type 2 diabetes. Methods: We enrolled 287 patients with type 2 diabetes, who were classified into normoalbuminuria $(n=144)$, microalbuminuria $(n=94)$, or macroalbuminuria $(n=49)$ groups based on their urine albumin to creatinine ratios, along with 42 healthy controls. We assessed 13 biomarkers, including transferrin (Tf), immunoglobulin $\mathrm{G}(\mathrm{IgG})$, podocalyxin (PCX), neutrophil gelatinase-associated lipocalin (NGAL), N-acetyl-beta-glucosaminidase (NAG), $\alpha$-1microglobulin ( $\alpha 1 M G$ ), 8-hydroxy-deoxyguanosine (8-OHdG), tumor necrosis factor-alpha (TNF- $\alpha$ ), and interleukin-18 (IL-18) in urine samples, along with cystatin C (CysC), total bilirubin (TBIL) , and uric acid (UA) in sera samples, to evaluate their diagnostic roles. From the measurements, the blood neutrophil to lymphocyte ratio (NLR) was also calculated.Results: Urinary Tf, IgG, NGAL, and TNF- $\alpha$ were significantly related to the UACR. We calculated the area under the receiver operating characteristic curves (ROC AUC) and found that urinary IgG (0.894), NGAL (0.875), Tf (0.861), TNF- $\alpha$ (0.763), and the combinations of urinary Tf $+\lg G+$ TNF- $\alpha+$ NGAL (0.922) showed good diagnostic value for early-stage DN. Conclusions: Urinary Tf, IgG , NGAL, and TNF- $\alpha$, and the combination of all four biomarkers demonstrated excellent diagnostic value for early-stage DN in patients with type 2 diabetes. 
1 The role of serum and urinary biomarkers in the diagnosis

2 of early diabetic nephropathy in patients with type 2

3 diabetes

4

5 Deyuan Zhang 1,2, Shandong Ye Corresp., 1,3, Tianrong Pan ${ }^{2}$

$7 \quad{ }^{1}$ School of Medicine, Shandong University, Jinan, Shandong, China

$8{ }^{2}$ Department of Endocrinology, The Second Affiliated Hospital of Anhui Medical University, He

9 fei, Anhui, China

$10{ }^{3}$ Department of Endocrinology, Anhui Provincial Hospital, Hefei, Anhui, China

Corresponding Author:

14 Shandong Ye

15 No. 17 Lujiang Road, Hefei, Anhui 230001, China.

16 Email address: yesd196406@163.com 


\section{Abstract}

19 Background: Previous studies have shown that a variety of biomarkers are closely related to the occurrence and development of early-stage diabetic nephropathy (DN) in patients. The aim of this study was to evaluate the role of multiple sera and urinary biomarkers in the diagnosis of early-stage $\mathrm{DN}$ in patients with type 2 diabetes.

Methods: We enrolled 287 patients with type 2 diabetes, who were classified into

24 normoalbuminuria $(n=144)$, microalbuminuria $(n=94)$, or macroalbuminuria $(n=49)$ groups based on their urine albumin to creatinine ratios, along with 42 healthy controls. We assessed 13 biomarkers, including transferrin (Tf), immunoglobulin $\mathrm{G}(\mathrm{IgG})$, podocalyxin (PCX), neutrophil gelatinase-associated lipocalin (NGAL), N-acetyl-beta-glucosaminidase $\quad(N A G), \quad \alpha-1-$ microglobulin $(\alpha 1 \mathrm{MG}), 8$-hydroxy-deoxyguanosine $(8-\mathrm{OHdG})$, tumor necrosis factor-alpha (TNF- $\alpha$ ), and interleukin-18 (IL-18) in urine samples, along with cystatin C (CysC), total bilirubin (TBIL), and uric acid (UA) in sera samples, to evaluate their diagnostic roles. From the measurements, the blood neutrophil to lymphocyte ratio (NLR) was also calculated.

Results: Urinary Tf, IgG, NGAL, and TNF- $\alpha$ were significantly related to the UACR. We calculated the area under the receiver operating characteristic curves (ROC AUC) and found that urinary IgG (0.894), NGAL (0.875), Tf (0.861), TNF- $\alpha$ (0.763), and the combinations of urinary Tf + IgG + TNF- $\alpha+$ NGAL (0.922) showed good diagnostic value for early-stage DN.

Conclusions: Urinary Tf, IgG, NGAL, and TNF- $\alpha$, and the combination of all four biomarkers demonstrated excellent diagnostic value for early-stage DN in patients with type 2 diabetes. 
39

40

41

4 43

44

45

46

47

48

49

50

51

52

60

\section{Materials \& Methods} diagnosis of early-stage DN. early-stage DN.

Diabetic nephropathy (DN), also known as diabetic kidney disease, is a common and severe microvascular complication of type 2 diabetes that can result in end-stage kidney disease. With the increasing incidence of type 2 diabetes worldwide, DN has become a global health concern ${ }^{1}$. The effective treatment of DN requires glycemic control and antihypertensive measures ${ }^{1}$. It is important to identify DN during its early stages as prompt treatment can reduce the medical and economic burden of this disease ${ }^{2,3}$.

Currently, microalbuminuria is the most widely investigated biomarker for the diagnosis of DN. However, its diagnostic value in early-stage DN is limited as renal injury commonly precedes proteinuria. In a previous study, Perkins et al. demonstrated that only $52.2 \%$ of patients with advanced-stage DN presented with proteinuria during a 12-year follow-up study ${ }^{4}$. In another study, only $33 \%$ of patients with type 1 diabetes having confirmed microalbuminuria developed clinically-apparent kidney disease ${ }^{5}$. Other studies have reaffirmed these findings, demonstrating that a considerable portion of diabetic patients with renal dysfunction do not have proteinuria ${ }^{6-8}$. Therefore, there is a dire need for more sensitive and specific biomarkers for the

It has been well-established that glomerular damage, tubular injury, inflammatory responses, and oxidative stress contribute to the development of diabetic kidney disease ${ }^{9}$. In this study, we evaluated 13 serum and urinary biomarkers involved in glomerular damage, tubular injury, inflammation, and oxidative stress, for their potential application in the diagnosis of

PeerJ reviewing PDF | (2018:12:33519:3:0:NEW 30 Apr 2019) 


\section{Patients}

63 In this cross-sectional study, 287 patients with type 2 diabetes (according to the 1999 WHO

64 criteria) who were hospitalized in the Department of Endocrinology, Second Affiliated Hospital

65 of Anhui Medical University between January 2018 and March 2018, were selected. In the same

66 period, 42 healthy medical volunteers from the Health Management Center of our hospital were

67 selected as the healthy controls. The collection of specimens was completed by the nursing staff,

68 and the samples were submitted to the clinical laboratory of our hospital for analysis. The 287

69 enrolled patients with type 2 diabetes who were classified as normoalbuminuric $(\mathrm{n}=144)$,

70 microalbuminuric $(n=94)$, and macroalbuminuric $(n=49)$ based on their urine albumin to

71 creatinine ratios (UACR) of $<30 \mathrm{mg} / \mathrm{g}, 30-300 \mathrm{mg} / \mathrm{g}$, and $>300 \mathrm{mg} / \mathrm{g}$, respectively.

72 Patients with the following conditions were excluded from this study: severe cardiac,

73 liver, and pancreatic diseases; primary glomerulonephritis or kidney diseases caused by

74 secondary conditions other than diabetes; infection, malignancies, or autoimmune disease; and

75 recent acute diabetic complications including ketoacidosis, hyperosmolar nonketotic diabetic

76 coma, and lactic acidosis. In addition to the above diseases, the healthy controls were free of

77 hypertension, hyperlipidemia, hyperuricemia, and hematological diseases. All patients enrolled

78 in this study provided oral informed consent before the study was conducted. The research

79 followed the tenets of the Declaration of Helsinki and was approved by the Medical Ethics

80 Committee of Anhui Medical University (Ethical Application Ref: 2017038).

81

\section{Data collection}

83 Demographic and clinical parameters, including gender, age, duration of diabetes, blood 84 pressure, height, body weight, body mass index (BMI), and fundus lesions, were collected. 
85 Fasting blood samples were drawn, and hemoglobin A1c (HbA1c) was measured using the HA-

$868160 \mathrm{HbAlc}$ analyzer (Arkray KDK, Japan). Fasting blood glucose (FBG), total cholesterol

87 (TC), triglyceride (TG), low-density lipoprotein (LDL), total bilirubin (TBIL), serum creatinine

88 (Scr), cystatin $\mathrm{C}$ (CysC), uric acid (UA), neutrophil count (NC), and lymphocyte count (LC)

89 were measured using UniCel Dxc 800 biochemical analyzer (Beckman Coulter, Brea, CA, USA).

90 The neutrophil to lymphocyte ratio (NLR) and estimated glomerular filtration rate (eGFR) were

91 calculated. The eGFR was calculated using the CKD-Epi formula ${ }^{10}$. The first midstream urine in

92 the morning was collected in a sterile cup and stored at $-80^{\circ} \mathrm{C}$ for analysis of urinary albumin

93 (UAlb), transferrin (Tf), N-acetyl-beta-glucosaminidase (NAG), immunoglobulin G (IgG), and

94 alpha-1-microglobulin ( $\alpha 1 \mathrm{MG})$ using an immunonephelometric assay with the BN2 analyzer

95 (Siemens Healthcare Diagnostics, Deerfield, IL, USA). The picric acid method was used for 96 determining urinary creatinine (Ucr) levels, while urinary podocalyxin (PCX), neutrophil 97 gelatinase-associated lipocalin (NGAL), 8-hydroxy-deoxyguanosine (8-OHdG), tumor necrosis 98 factor alpha (TNF- $\alpha$ ), and interleukin-18 (IL-18) were measured with a commercial enzyme99 linked immunosorbent assay kits (Elabscience Biotechnology, Wuhan, Hubei, China). All 100 biomarker specimens were collected and tested at our hospital. In order to eliminate the effect of 101 urine concentration or dilution on the results, all measurements from the urine were presented as 102 the ratio of the measured values to Ucr. For values $<\mathrm{X}$ (where $\mathrm{X}$ is a number) in the raw data 103 which were values that were below the detection threshold of our method used to measure 104 biomarker concentrations, the threshold value were used as the estimate in the analysis. 
108 Data were analyzed using SPSS software version 16.0 (IBM, Chicago, IL, USA). Continuous

109 variables with normal distribution were expressed as mean \pm standard deviation, and non-

110 normally distributed data were expressed as median (25th percentile, 75th percentile).

111 Differences among the groups were analyzed by the one-way analysis of variance (ANOVA),

112 and comparisons between two groups were analyzed by the Student's t-test. Categorical

113 variables were compared using the Wilcoxon rank-sum test or chi-squared test. The multivariate

114 linear regression analysis was used to analyze the correlation between the different biomarkers

115 and UACR. Correlation with UACR was defined if the t-test for the partial regression

116 coefficients were statistically significant in the regression models.Receiver operating

117 characteristic curve (ROC) analysis were used to assess the diagnostic values of each biomarker

118 and the combination of biomarkers,and also to test whether the null hypothesis that biomarker

119 were not indicative of early-stage DN was true. The area under the curve (AUC), sensitivity, and

120 specificity of parameters were calculated based on the ROC.The ROC curve was the plot of all

121 possible pairs of sensitivity and specificity values obtained by scrutinizing all possible values of

122 the cut off. The optimal cut off point with relevant sensitivity and specificity was found where

123 the Youden's index was maximum ${ }^{11} . \mathrm{P}<0.05$ was considered statistically significant.

\section{Results}

127 The parameters of each group are shown in Table 1. The Tf, IgG, PCX, CysC, NAG, $\alpha 1 \mathrm{MG}, 8$ -

$128 \mathrm{OHdG}$, IL-18, and UA levels of patients in the macroalbuminuria group were higher than those 129 in the other three groups $(P<0.05$ for UA, $P<0.001$ for other parameters). The NGAL, TNF- $\alpha$, 130 and NLR levels in the macroalbuminuria group were higher than those of the healthy control and 
131 normoalbuminuria groups $(P<0.001)$. The NLR levels in the macroalbuminuria group were

132 higher than those in the microalbuminuria group $(P<0.05)$. TBIL in the macroalbuminuria group 133 was lower than the normoalbuminuria and microalbuminuria groups $(P<0.001)$. The Tf, IgG, 134 PCX, NGAL, NAG, $\alpha 1 \mathrm{MG}, 8-\mathrm{OHdG}, \mathrm{TNF}-\alpha$, IL-18, and NLR levels of patients in the 135 microalbuminuria group were higher than the healthy controls and normoalbuminuria groups 136 ( $P<0.05$ for NLR, $P<0.001$ for other parameters). The Tf, PCX, NGAL, NAG, $\alpha 1 \mathrm{MG}, \mathrm{TBIL}$, and 137 IL-18 levels in patients with normoalbuminuria were higher than the healthy controls $(P<0.001$ 138 or $P<0.05)$.

139 The multivariate linear regression was performed for the biomarkers correlated with 140 UACR, and adjustments were made to account for risk factors, such as gender, age, diabetes 141 duration, HbA1c, eGFR, LDL, and systolic blood pressure and diastolic blood pressure. Urinary $142 \mathrm{Tf}, \mathrm{IgG}, \mathrm{NGAL}$, and TNF- $\alpha$ were found to be significantly related to the UACR in patients with 143 type 2 diabetes $(\mathrm{P}<0.001)$ (Table 2$)$. The diagnostic value of urinary Tf, IgG, NGAL, and TNF- $\alpha$ 144 were analyzed by ROC curves (Fig. 1) and the results showed that, in terms of AUC values, IgG > 145 NGAL $>$ Tf $>$ TNF- $\alpha$; in terms of sensitivity, NGAL $>$ IgG $>$ Tf $>$ TNF- $\alpha$; and in terms of 146 specificity, $\mathrm{IgG}>\mathrm{Tf}>\mathrm{TNF}-\alpha>\mathrm{NGAL}(\mathrm{P}<0.001)$ (Table 3).

147 The diagnostic value of the combination of urinary Tf, IgG, NGAL, and TNF- $\alpha$ were 148 analyzed with ROC curves(Fig. 2). The results showed that the combination of urinary Tf, IgG, 149 NGAL, and TNF- $\alpha$ had the highest AUC when compared with the individual 150 biomarkers $(\mathrm{P}<0.001)$ (Table 3).

151

\section{Discussion}

$153 \mathrm{DN}$ is a chronic and progressive kidney disease characterized by microalbuminuria and 
154 decreased glomerular filtration rates, which can eventually lead to end-stage renal disease and

155 death. However, its early diagnosis and intervention could allow for the prompt treatment of DN,

156 which would delay the pathological progression of the disease. In this study, we evaluated

157 several potential biomarkers for their diagnostic potential for detecting early-stage DN. The

158 primary finding of this study was that urinary $\mathrm{Tf}, \mathrm{IgG}, \mathrm{NGAL}$, and TNF- $\alpha$ levels, both

159 individually and in combination, are valuable biomarkers for assessing early-stage DN in 160 patients with type 2 diabetes.

161 While some highly sensitive and specific biomarkers were previously identified for the

162 diagnosis of $\mathrm{DN}^{12}$, this study covered multiple parameters involved in the four major

163 mechanisms of DN and evaluated the diagnostic value of them individually and in combination.

164 The pathogenic mechanisms of DN include glomerular damage, tubular injury, inflammation, 165 and oxidative stress 13,14. Therefore, biomarkers involved in these processes may potentially 166 serve as clinically-relevant diagnostic parameters of $\mathrm{DN}^{15}$.

167 Glomerular damage leads to increased urinary excretion of plasma proteins, such as Tf, 168 IgG, and PCX ${ }^{16}$. Tf is a plasma protein with a similar molecular weight as albumin. However, 169 Tf is less anionic than albumin, which makes it more easily filtered via the glomerular barrier. 170 Previous studies found that high urinary Tf levels in type 2 diabetic patients with and without 171 microalbuminuria 17,18 . A five-year follow-up study found that elevated urinary Tf levels could 172 be used to predict the progression to microalbuminuria in patients with type 2 diabetes ${ }^{19}$. While 173 urinary IgG is also elevated in some patients prior to microalbuminuria ${ }^{18}$. This suggests that 174 urinary IgG levels may be a clinically-relevant biomarker for the early assessment and diagnosis 175 of DN in patients with type 2 diabetes ${ }^{19}$. In the current study, we found that urinary Tf and IgG 176 may be used alone or in combination with other biomarkers for the diagnosis of early-stage DN. 
177 PCX, a negatively charged sialoglycoprotein, is expressed in podocytes and plays an essential

178 role in maintaining the function of glomerular podocytes ${ }^{20}$. Urinary PCX levels were found to be 179 associated with UACR levels and were also increased in diabetic patients with 180 normoalbuminuria ${ }^{21}$. While PCX may be an excellent marker for podocyte injury ${ }^{12}$, it is not 181 considered to be an excellent diagnostic parameter for DN by the current study.

182 Renal tubular injury is a critical characteristic of DN ${ }^{22}$. Urinary tubular injury markers 183 may increase in diabetic patients, even before the onset of microalbuminuria ${ }^{23}$. NGAL, also 184 named lipocalin-2, is a secretory protein that is released during renal injury and serves as a 185 marker of acute kidney injury ${ }^{24}$. $\mathrm{CysC}$, an inhibitor of the cysteine protease, is freely filtered 186 through the glomeruli and reabsorbed by the proximal tubule ${ }^{25}$. Previous studies demonstrated 187 that urinary NGAL and CysC were higher in diabetic patients and positively associated with 188 UACR ${ }^{26-28}$. Our study found that urinary NGAL was increased in diabetic patients with 189 microalbuminuria, as well as diabetic patients with normoalbuminuria when compared with the 190 healthy controls. However, CysC was only higher in patients with macroalbuminuria. Similarly, 191 another study showed that $\mathrm{CysC}$ was only elevated in advanced-stage diabetic kidney disease 192 and remained at normal levels during the early stages of the disease ${ }^{29}$. NAG is a lysosomal 193 enzyme expressed in proximal tubules and was previously found to be a sensitive marker of 194 tubular injury ${ }^{30}$. Urinary NAG was increased in type 2 diabetic patients compared to non195 diabetic subjects, with higher levels in patients with microalbuminuria than those with 196 normoalbuminuria levels ${ }^{31,32}$. The $\alpha 1 \mathrm{MG}$ protein is freely filtered and reabsorbed through renal 197 tubules. Urinary $\alpha 1 \mathrm{MG}$ was also increased before the onset of microalbuminuria ${ }^{33,34}$. Although 198 we failed to identify NAG and $\alpha 1 M G$ as early diagnostic markers, NAG was significantly 199 elevated in the macroalbuminuria and microalbuminuria groups. Interestingly, $\alpha 1 \mathrm{MG}$ levels 
200 differed among the four groups, suggesting that these two parameters might play a role in the 201 assessment of disease severity and progression.

202 Oxidative stress is one of the mechanisms for $\mathrm{DN}^{35} \cdot 8-\mathrm{OHdG}$ is a stable oxidative 203 product and a marker of DNA damage ${ }^{36}$. Urinary $8-\mathrm{OHdG}$ was increased in patients with type 2 204 diabetes and was found to be a marker for monitoring the progression of $\mathrm{DN}{ }^{37}$, 38. However, 205 another study found that urinary 8-OHdG levels were similar in patients with DN and diabetic 206 patients with normoalbuminuria ${ }^{39}$. In our study, although elevated urinary 8-OHdG levels were 207 found in in the macroalbuminuria and microalbuminuria groups, urinary 8-OHdG was not 208 considered to be a valuable diagnostic biomarker for early-stage DN. Bilirubin is an endogenous 209 antioxidant, and low serum bilirubin levels are considered to be a risk factor for diabetic kidney 210 damage ${ }^{40}$. UA was reported to be associated with oxidative stress and endothelial dysfunction ${ }^{41}$, 211 and was previously considered as a risk factor for $\mathrm{DN}{ }^{42}$. However, another study found that UA 212 levels were not associated with the progression of $\mathrm{DN}{ }^{43}$. In the current study, TBIL and UA 213 levels in the macroalbuminuria group differed from the normoalbuminuria and microalbuminuria 214 groups, but there were no differences between the normoalbuminuria and microalbuminuria 215 groups. Therefore, these two biomarkers were not considered as potential candidates for the 216 detection of early-stage DN. However, they may be useful for assessing the severity of renal 217 damage.

218 DN is a low-grade inflammatory disease ${ }^{13}$, yet this study revealed a strong connection 219 with TNF- $\alpha$, which is a well-known inflammatory cytokine associated with renal injury ${ }^{44}$. 220 Compared to the normoalbuminuria group, a $90 \%$ increase in urinary TNF- $\alpha$ excretion was 221 present in type 2 diabetic patients with microalbuminuria, which was further correlated with the 222 UACR ${ }^{45}$. Urinary IL-18 was positively associated with urine protein excretion and may be a 
223 predictive factor for assessing the progression of DN 46, 47, yet one study disagreed with this

224 finding ${ }^{48}$. NLR is a novel inflammatory marker known to be increased in patients with type 2

225 diabetes when compared with the healthy controls. NLR was also higher in patients with

226 microalbuminuria group when compared with the normoalbuminuria group ${ }^{49,50}$. Our study

227 found elevated levels of TNF- $\alpha$, NLR, and IL-18 in patients with microalbuminuria and

228 macroalbuminuria that were significantly higher than those detected in the healthy controls and 229 type 2 diabetic patients with normoalbuminuria. However, only TNF- $\alpha$ was identified as a 230 candidate biomarker for the detection of early-stage DN.

231 Using the ROC analysis, four biomarkers (urinary Tf, IgG, NGAL, and TNF- $\alpha$ ) showed

232 diagnostic value in terms of high AUC, in type 2 diabetic patients with DN. Urinary IgG showed 233 a high specificity of $92.3 \%$, yet the sensitivity was relatively low. Similarly, urinary NGAL was 234 highly sensitive but suffered from its low specificity. Further analyses revealed the combination 235 of these four biomarkers had better diagnostic value, in terms of AUC value,s than those of the 236 biomarkers individually. The AUC was as high as 0.922 for the combined biomarkers, indicating 237 a superior value in the detection of early-stage DN.

\section{Conclusions}

240 In conlusion, this study found that urinary Tf, IgG, NGAL, and TNF- $\alpha$ levels may be used as 241 biomarkers for the diagnosis of early-stage DN in patients with type 2 diabetes. However, the 242 combination of these four biomarkers showed higher sensitivity and specificity in comparison to

243 the biomarkers when used individually. The four biomarkers assessed in this study are involved 244 in glomerular damage, tubular injury, and inflammation, respectively, which represents different 245 aspects of the pathogenesis of DN. The combined use of these novel biomarkers may improve 
246 the detection of early-stage DN. However, further studies using larger sample sizes should be

247 performed to establish the diagnostic value of other biomarkers for detection of early-stage DN.

248

249 Acknowledgements

250 My deepest gratitude goes first and foremost to professor Shandong Ye.

251

252 References

253 1. Wan Q, Xu Y, Dong E. Diabetic nephropathy research in China: Data analysis and review

254 from the National Natural Science Foundation of China. J Diabetes. 2015;7:307-314. doi: $255 \quad 10.1111 / 1753-0407.12265$.

256 2. Tziomalos K, Athyros VG. Diabetic Nephropathy: New Risk Factors and Improvements in 257 Diagnosis. Rev Diabet Stud. 2015;12:110-118. doi: 10.1900/RDS.2015.12.110.

258 3. Gudehithlu KP, Hart PD, Vernik J, Sethupathi P, Dunea G, Arruda JAL, Singh AK. 259 Peptiduria: a potential early predictor of diabetic kidney disease. Clin Exp Nephrol. 2018. doi: $26010.1007 / \mathrm{s} 10157-018-1620-0$.

261 4. Perkins BA, Ficociello LH, Roshan B, Warram JH, Krolewski AS. In patients with type 1 262 diabetes and new-onset microalbuminuria the development of advanced chronic kidney disease 263 may not require progression to proteinuria. Kidney Int. 2010;77:57-64. doi: 10.1038/ki.2009.399.

264 5. Rossing P, Hougaard P, Parving HH. Progression of microalbuminuria in type 1 diabetes: ten265 year prospective observational study. Kidney Int. 2005;68:1446-1450. doi: 10.1111/j.1523266 1755.2005.00556.x. 
267 6. An JH, Cho YM, Yu HG, Jang HC, Park KS, Kim SY, Lee HK. The clinical characteristics of 268 normoalbuminuric renal insufficiency in Korean type 2 diabetic patients: a possible early stage 269 renal complication. J Korean Med Sci. 2009;24 Suppl:S75-81. doi: $270 \quad$ 10.3346/jkms.2009.24.S1.S75.

271 7. Kramer HJ, Nguyen QD, Curhan G, Hsu CY. Renal insufficiency in the absence of 272 albuminuria and retinopathy among adults with type 2 diabetes mellitus. JAMA. 2003;289:3273273 3277. doi: 10.1001/jama.289.24.3273.

274 8. Zachwieja J, Soltysiak J, Fichna P, Lipkowska K, Stankiewicz W, Skowronska B, Kroll 275 P, Lewandowska-Stachowiak M. Normal-range albuminuria does not exclude nephropathy in 276 diabetic children. Pediatr Nephrol. 2010;25:1445-1451. doi: 10.1007/s00467-010-1443-z.

277 9. Gurley SB, Ghosh S, Johnson SA, Azushima K, Sakban RB, George SE, Maeda M, Meyer 278 TW, Coffman TM. Inflammation and Immunity Pathways Regulate Genetic Susceptibility to 279 Diabetic Nephropathy. Diabetes. 2018. doi: 10.2337/db17-1323.

280 281 282 283 284 285 286 287 288
10. Levey AS, Stevens LA, Schmid CH, Zhang YL, Castro AF, 3rd, Feldman HI, Kusek JW, Eggers P, Van Lente F, Greene T, Coresh J; CKD-EPI (Chronic Kidney Disease Epidemiology Collaboration). A new equation to estimate glomerular filtration rate. Ann Intern Med. 2009;150(9):604-12.

11. Lai CY, Tian L, Schisterman EF. Exact confidence interval estimation for the Youden index and its corresponding optimal cut-point.Comput Stat Data Anal. 2012 May 1;56(5):1103-1114.

doi: 10.1016/j.csda.2010.11.023

12. Hara M, Yamagata K, Tomino Y, Ogasawara S, Kurosawa H, Sekine S, Yan K. Urinary podocalyxin is an early marker for podocyte injury in patients with diabetes: establishment of a 
289 highly sensitive ELISA to detect urinary podocalyxin. Diabetologia. 2012;55:2913-2919. doi: $290 \quad 10.1007 / \mathrm{s} 00125-012-2661-7$.

291 13. Wada J, Makino H. Inflammation and the pathogenesis of diabetic nephropathy. Clin Sci 292 (Lond). 2013;124:139-152. doi: 10.1042/CS20120198.

293 14. Arora MK, Singh UK. Molecular mechanisms in the pathogenesis of diabetic nephropathy: 294 an update. Vascul Pharmacol. 2013;58:259-271. doi: 10.1016/j.vph.2013.01.001.

295 15. Gluhovschi C, Gluhovschi G, Petrica L, Timar R3, Velciov S1, Ionita I4, Kaycsa A5, Timar 296 B3. Urinary Biomarkers in the Assessment of Early Diabetic Nephropathy. J Diabetes Res. 297 2016;2016:4626125. doi: 10.1155/2016/4626125.

298 16. Currie G, McKay G, Delles C. Biomarkers in diabetic nephropathy: Present and future. 299 World J Diabetes. 2014;5:763-776. doi: 10.4239/wjd.v5.i6.763.

300 17. Kanauchi M, Akai Y, Hashimoto T. Transferrinuria in type 2 diabetic patients with early 301 nephropathy and tubulointerstitial injury. Eur J Intern Med. 2002;13:190-193. doi.

302 18. Narita T, Sasaki H, Hosoba M, Miura T, Yoshioka N, Morii T, Shimotomai T, Koshimura 303 J, Fujita H, Kakei M, Ito S. Parallel increase in urinary excretion rates of immunoglobulin G, 304 ceruloplasmin, transferrin, and orosomucoid in normoalbuminuric type 2 diabetic patients. 305 Diabetes Care. 2004;27:1176-1181. doi.

306 19. Narita T, Hosoba M, Kakei M, Ito S. Increased urinary excretions of immunoglobulin g, 307 ceruloplasmin, and transferrin predict development of microalbuminuria in patients with type 2 308 diabetes. Diabetes Care. 2006;29:142-144. doi. 
309 20. Lee SY, Choi ME. Urinary biomarkers for early diabetic nephropathy: beyond albuminuria.

310 Pediatr Nephrol. 2015;30:1063-1075. doi: 10.1007/s00467-014-2888-2.

311 21. Shoji M, Kobayashi K, Takemoto M, Sato Y, Yokote K. Urinary podocalyxin levels were 312 associated with urinary albumin levels among patients with diabetes. Biomarkers. 2016;21:164313 167. doi: 10.3109/1354750X.2015.1118551.

314 22. Kumar D, Robertson S, Burns KD. Evidence of apoptosis in human diabetic kidney. Mol 315 Cell Biochem. 2004;259:67-70. doi.

316 23. Russo LM, Sandoval RM, Campos SB, Molitoris BA, Comper WD, Brown D. Impaired 317 tubular uptake explains albuminuria in early diabetic nephropathy. J Am Soc Nephrol. 318 2009;20:489-494. doi: 10.1681/ASN.2008050503.

319 24. Bolignano D, Lacquaniti A, Coppolino G, Donato V, Fazio MR, Nicocia G, Buemi M. 320 Neutrophil gelatinase-associated lipocalin as an early biomarker of nephropathy in diabetic 321 patients. Kidney Blood Press Res. 2009;32:91-98. doi: 10.1159/000209379.

322 25. Jeon YK, Kim MR, Huh JE, Mok JY, Song SH, Kim SS, Kim BH, Lee SH, Kim YK, Kim IJ. 323 Cystatin C as an early biomarker of nephropathy in patients with type 2 diabetes. J Korean Med 324 Sci. 2011;26:258-263. doi: 10.3346/jkms.2011.26.2.258.

325 26. Assal HS, Tawfeek S, Rasheed EA, El-Lebedy D, Thabet EH. Serum cystatin C and tubular 326 urinary enzymes as biomarkers of renal dysfunction in type 2 diabetes mellitus. Clin Med 327 Insights Endocrinol Diabetes. 2013;6:7-13. doi: 10.4137/CMED.S12633. 
328

329

330

331

332

333

334 335

336

337

338

339

340

341

342 343

344

345

346

347

348

349

27. Mahfouz MH, Assiri AM, Mukhtar MH. Assessment of Neutrophil Gelatinase-Associated Lipocalin (NGAL) and Retinol-Binding Protein 4 (RBP4) in Type 2 Diabetic Patients with Nephropathy. Biomark Insights. 2016;11:31-40. doi: 10.4137/BMI.S33191.

28. Rao X, Wan M, Qiu C, Jiang C. Role of cystatin C in renal damage and the optimum cut-off point of renal damage among patients with type 2 diabetes mellitus. Exp Ther Med. 2014;8:887892. doi: 10.3892/etm.2014.1815.

29. Ogawa Y, Goto T, Tamasawa N, Matsui J, Tando Y, Sugimoto K, Tomotsune K, Kimura M, Yasujima M, Suda T. Serum cystatin C in diabetic patients. Not only an indicator for renal dysfunction in patients with overt nephropathy but also a predictor for cardiovascular events in patients without nephropathy. Diabetes Res Clin Pract. 2008;79:357-361. doi: 10.1016/j.diabres.2007.09.016.

30. Bazzi C, Petrini C, Rizza V, Arrigo G, Napodano P, Paparella M, D'Amico G. Urinary Nacetyl-beta-glucosaminidase excretion is a marker of tubular cell dysfunction and a predictor of outcome in primary glomerulonephritis. Nephrol Dial Transplant. 2002;17:1890-1896. doi.

31. Sheira G, Noreldin N, Tamer A, Saad M. Urinary biomarker N-acetyl-beta-Dglucosaminidase can predict severity of renal damage in diabetic nephropathy. J Diabetes Metab Disord. 2015;14:4. doi: 10.1186/s40200-015-0133-6.

32. Bouvet BR, Paparella CV, Arriaga SM, Monje AL, Amarilla AM, Almara AM. Evaluation of urinary N-acetyl-beta-D-glucosaminidase as a marker of early renal damage in patients with type 2 diabetes mellitus. Arq Bras Endocrinol Metabol. 2014;58:798-801. doi.

33. Shore N, Khurshid R, Saleem M. Alpha-1 microglobulin: a marker for early detection of tubular disorders in diabetic nephropathy. J Ayub Med Coll Abbottabad. 2010;22:53-55. doi. 
350 34. Hong CY, Hughes K, Chia KS, Ng V, Ling SL. Urinary alpha1-microglobulin as a marker of

351 nephropathy in type 2 diabetic Asian subjects in Singapore. Diabetes Care. 2003;26:338-342.

352 doi.

353 35. Forbes JM, Coughlan MT, Cooper ME. Oxidative stress as a major culprit in kidney disease

354 in diabetes. Diabetes. 2008;57:1446-1454. doi: 10.2337/db08-0057.

355 36. Valavanidis A, Vlachogianni T, Fiotakis C. 8-hydroxy-2' -deoxyguanosine (8-OHdG): A

356 critical biomarker of oxidative stress and carcinogenesis. J Environ Sci Health C Environ

357 Carcinog Ecotoxicol Rev. 2009;27:120-139. doi: 10.1080/10590500902885684.

358

359

360

361

362

363

364

365

366

367

368

369

370

371
37. Waris S, Winklhofer-Roob BM, Roob JM, Fuchs S, Sourij H, Rabbani N, Thornalley PJ. Increased DNA dicarbonyl glycation and oxidation markers in patients with type 2 diabetes and link to diabetic nephropathy. J Diabetes Res. 2015;2015:915486. doi: 10.1155/2015/915486.

38. Hinokio Y, Suzuki S, Hirai M, Suzuki C, Suzuki M, Toyota T. Urinary excretion of 8-oxo-7, 8-dihydro-2'-deoxyguanosine as a predictor of the development of diabetic nephropathy. Diabetologia. 2002;45:877-882. doi: 10.1007/s00125-002-0831-8.

39. Serdar M, Sertoglu E, Uyanik M, Tapan S, Akin K, Bilgi C, Kurt I. Comparison of 8hydroxy-2'-deoxyguanosine $(8-\mathrm{OHdG})$ levels using mass spectrometer and urine albumin creatinine ratio as a predictor of development of diabetic nephropathy. Free Radic Res. 2012;46:1291-1295. doi: 10.3109/10715762.2012.710902.

40. Okada H, Fukui M, Tanaka M, Matsumoto S, Kobayashi K, Iwase H, Tomiyasu K, Nakano K, Hasegawa G, Nakamura N. Low serum bilirubin concentration is a novel risk factor for the development of albuminuria in patients with type 2 diabetes. Metabolism. 2014;63:409-414. doi: 10.1016/j.metabol.2013.11.011.

PeerJ reviewing PDF | (2018:12:33519:3:0:NEW 30 Apr 2019) 
372 41. Jalal DI, Maahs DM, Hovind P, Nakagawa T. Uric acid as a mediator of diabetic 373 nephropathy. Semin Nephrol. 2011;31:459-465. doi: 10.1016/j.semnephrol.2011.08.011.

374 42. Jalal DI, Rivard CJ, Johnson RJ, Maahs DM, McFann K, Rewers M, Snell-Bergeon JK. 375 Serum uric acid levels predict the development of albuminuria over 6 years in patients with type 3761 diabetes: findings from the Coronary Artery Calcification in Type 1 Diabetes study. Nephrol 377 Dial Transplant. 2010;25:1865-1869. doi: 10.1093/ndt/gfp740.

378 43. Ahola AJ, Sandholm N, Forsblom C, Harjutsalo V, Dahlström E, Groop PH; FinnDiane 379 Study Group. The serum uric acid concentration is not causally linked to diabetic nephropathy in 380 type 1 diabetes. Kidney Int. 2017;91:1178-1185. doi: 10.1016/j.kint.2016.11.025.

381 44. Navarro JF, Mora C, Muros M, Garcia J. Urinary tumour necrosis factor-alpha excretion 382 independently correlates with clinical markers of glomerular and tubulointerstitial injury in type 3832 diabetic patients. Nephrol Dial Transplant. 2006;21:3428-3434. doi: 10.1093/ndt/gfl469.

384 45. Navarro JF, Mora C, Gomez M, Muros M, Lopez-Aguilar C, Garcia J. Influence of renal 385 involvement on peripheral blood mononuclear cell expression behaviour of tumour necrosis 386 factor-alpha and interleukin-6 in type 2 diabetic patients. Nephrol Dial Transplant. 2008;23:919387 926. doi: 10.1093/ndt/gfm674.

46. Liu F, Guo J, Zhang Q, Liu D, Wen L, Yang Y, Yang L, Liu Z. The Expression of 389 Tristetraprolin and Its Relationship with Urinary Proteins in Patients with Diabetic Nephropathy. 390 PLoS One. 2015;10:e0141471. doi: 10.1371/journal.pone.0141471.

391 47. Nakamura A, Shikata K, Hiramatsu M, Nakatou T, Kitamura T, Wada J, Itoshima T, Makino

392 H. Serum interleukin-18 levels are associated with nephropathy and atherosclerosis in Japanese 393 patients with type 2 diabetes. Diabetes Care. 2005;28:2890-2895. doi. 
394 48. Nadkarni GN, Rao V, Ismail-Beigi F, Fonseca VA, Shah SV, Simonson MS, Cantley 395 L, Devarajan P, Parikh CR, Coca SG. Association of Urinary Biomarkers of Inflammation, 396 Injury, and Fibrosis with Renal Function Decline: The ACCORD Trial. Clin J Am Soc Nephrol. 397 2016;11:1343-1352. doi: 10.2215/CJN.12051115.

398 49. Huang W, Huang J, Liu Q, Lin F, He Z, Zeng Z, He L. Neutrophil-lymphocyte ratio is a 399 reliable predictive marker for early-stage diabetic nephropathy. Clin Endocrinol (Oxf). $400 \quad$ 2015;82:229-233. doi: 10.1111/cen.12576.

401 50. Khandare SA, Chittawar S, Nahar N, Dubey TN, Qureshi Z. Study of Neutrophil-lymphocyte 402 Ratio as Novel Marker for Diabetic Nephropathy in Type 2 Diabetes. Indian J Endocrinol Metab. 403 2017;21:387-392. doi: 10.4103/ijem.IJEM_476_16.

404

405 


\section{Table $\mathbf{1}$ (on next page)}

Clinical and laboratory characteristics of patients in the normoalbuminuria $(n=144)$, microalbuminuria $(n=94)$, macroalbuminuria $(n=49)$, and healthy control $(n=42)$ groups. 
1 Table 1. Clinical and laboratory characteristics of patients in the normoalbuminuria $(n=144)$,

2 microalbuminuria $(n=94)$, macroalbuminuria $(n=49)$, and healthy control $(n=42)$ groups.

\begin{tabular}{|c|c|c|c|c|c|c|}
\hline & $\begin{array}{l}\text { Healthy controls } \\
\qquad(\mathrm{n}=42)\end{array}$ & $\begin{array}{l}\text { Normoalbuminuria } \\
\qquad(\mathrm{n}=144)\end{array}$ & $\begin{array}{l}\text { Microalbuminuria } \\
\qquad(\mathrm{n}=94)\end{array}$ & $\begin{array}{l}\text { Macroalbuminuria } \\
\qquad(\mathrm{n}=49)\end{array}$ & $F / \chi^{2}$ & $p$-value \\
\hline Gender & & & & & 0.189 & 0.979 \\
\hline Male, n (\%) & $23(54.80)$ & $83(57.64)$ & $52(55.32)$ & $28(57.14)$ & & \\
\hline Female, n (\%) & $19(45.20)$ & $61(42.36)$ & $42(44.68)$ & $21(42.86)$ & & \\
\hline Age (years) & $54.33 \pm 14.974$ & $54.32 \pm 14.07$ & $55.49 \pm 15.26$ & $59.20 \pm 13.03^{\star *}$ & 3.674 & 0.013 \\
\hline $\operatorname{BMI}\left(\mathrm{kg} / \mathrm{m}^{2}\right)$ & $23.58 \pm 3.44$ & $25.51 \pm 3.56$ & $25.70 \pm 4.32$ & $25.45 \pm 3.58$ & 3.426 & 0.017 \\
\hline Duration (years) & - & $5.00(2.00,10.00)$ & $7.1(1.00,13.00)$ & $10.00(7.50,19.00)^{* * \#}$ & 18.904 & $<0.001$ \\
\hline Diabetic retinopathy, n (\%) & - & $31(21.53)$ & $32(34.04)^{*}$ & $35(71.43)^{* * \# \#}$ & 40.485 & $<0.001$ \\
\hline $\begin{array}{l}\text { Systolic blood pressure } \\
(\mathrm{mmHg})\end{array}$ & $122.55 \pm 10.31$ & $128.72 \pm 15.69^{\star}$ & $136.66 \pm 18.27 \star \star * *$ & $148.04 \pm 21.34 \star \star * * \#$ & 22.924 & $<0.001$ \\
\hline $\begin{array}{l}\text { Diastolic blood pressure } \\
(\mathrm{mmHg})\end{array}$ & $73.02 \pm 6.97$ & $78.25 \pm 9.76^{\star}$ & $80.20 \pm 10.54^{\star \star}$ & $81.96 \pm 12.76^{\star \star *}$ & 6.787 & $<0.001$ \\
\hline $\mathrm{FBG}(\mathrm{mmol} / \mathrm{L})$ & $4.89(4.58,5.31)$ & $7.45(5.99,9.73)^{\star \star}$ & $8.50(6.69,11.21)^{\star \star *}$ & $9.09(6.61,11.16)^{\star \star *}$ & 77.190 & 0.027 \\
\hline $\operatorname{Scr}(\mu \mathrm{mol} / \mathrm{L})$ & $57.50(48.50,77.00)$ & $71.00(56.25,85.00)^{\star}$ & $70.00(56.00,87.50)^{\star}$ & $\begin{array}{l}105.00(80.00 \\
149.00)^{\star \star * * \# \#}\end{array}$ & 55.409 & $<0.001$ \\
\hline $\mathrm{eGFR}\left(\mathrm{mL} / \mathrm{min} / 1.73 \mathrm{~m}^{2}\right)$ & $153.91 \pm 49.68$ & $127.14 \pm 41.00^{\star \star}$ & $122.97 \pm 43.31 \star \star$ & $79.33 \pm 44.53 \star \star * * \#$ & 23.755 & $<0.001$ \\
\hline $\mathrm{TC}(\mathrm{mmol} / \mathrm{L})$ & $4.45(3.64,5.12)$ & $4.50(3.92,5.03)$ & $4.64(4.02,5.52)$ & $4.97(4.09,5.78)^{*}$ & 6.634 & 0.085 \\
\hline $\mathrm{TG}(\mathrm{mmol} / \mathrm{L})$ & $1.16(0.81,1.89)$ & $1.38(0.95,2.12)$ & $1.49(1.03,2.65)^{\star}$ & $1.72(1.16,2.66)^{\star}$ & 9.907 & 0.019 \\
\hline $\mathrm{LDL}(\mathrm{mmol} / \mathrm{L})$ & $2.86 \pm 1.03$ & $2.82 \pm 0.84$ & $2.90 \pm 0.81$ & $3.06 \pm 1.04$ & 0.875 & 0.454 \\
\hline $\mathrm{HbA1c}$ & $5.38 \pm 0.44$ & $8.93 \pm 2.14^{\star \star}$ & $9.41 \pm 2.50 \star \star$ & $8.92 \pm 2.72^{\star \star}$ & 35.176 & $<0.001$ \\
\hline $\mathrm{Tf} / \mathrm{Ucr}(\mathrm{mg} / \mathrm{g})$ & $1.72(1.25,2.48)$ & $2.18(1.42,3.02)^{\star}$ & $5.79(3.12,8.77)^{\star \star * *}$ & $43.60(21.40,182.33)^{\star \star * * \# \#}$ & 199.600 & $<0.001$ \\
\hline $\operatorname{IgG} / \operatorname{Ucr}(\mathrm{mg} / \mathrm{g})$ & $4.44(3.00,6.59)$ & $5.23(3.57,6.61)$ & $12.85(8.55,18.43)^{\star \star * *}$ & $\begin{array}{l}106.19(41.28 \\
374.07)^{\star \star * * \#}\end{array}$ & 178.440 & $<0.001$ \\
\hline
\end{tabular}




\begin{tabular}{|c|c|c|c|c|c|c|}
\hline PCX/Ucr $(\mu \mathrm{g} / \mathrm{g})$ & $2.98(2.04,5.09)$ & $4.60(2.30,7.20)^{\star \star}$ & $6.69(3.16,13.16)^{\star \star * *}$ & $9.93(7.69,15.53)^{\star \star * * \#}$ & 72.919 & $<0.001$ \\
\hline NGAL $/ U c r(\mu \mathrm{g} / \mathrm{g})$ & $13.03(9.80,23.93)$ & $\begin{array}{l}20.15(12.37 \\
31.35)^{\star \star}\end{array}$ & $\begin{array}{l}67.09(38.39 \\
114.16)^{\star \star^{* *}}\end{array}$ & $60.44(27.09,106.00)^{\star \star * *}$ & 138.604 & $<0.001$ \\
\hline CysC (mg/L) & $0.72(0.61,0.89)$ & $0.72(0.59,0.90)$ & $0.79(0.60,1.00)$ & $1.12(0.81,1.90)^{\star \star * * \# \#}$ & 45.945 & $<0.001$ \\
\hline NAG/Ucr (U/g) & $10.19(6.52,13.41)$ & $13.56(8.24,18.80)^{\star \star}$ & $\begin{array}{l}25.26(14.46 \\
42.28)^{\star \star * *}\end{array}$ & $50.63(30.24,100.06)^{\star \star * * \#}$ & 144.974 & $<0.001$ \\
\hline$\alpha 1 \mathrm{MG} / \mathrm{Ucr}(\mathrm{mg} / \mathrm{g})$ & $8.51(5.75,14.78)$ & $11.88(7.98,18.46)^{\star \star}$ & $24.48(13.92,40.20)^{\star \star * *}$ & $77.56(34.82,121.76)^{\star \star * * \#}$ & 122.952 & $<0.001$ \\
\hline 8-OHdG/Ucr $(\mu \mathrm{g} / \mathrm{g})$ & $6.56 \pm 3.50$ & $7.36 \pm 4.42$ & $14.42 \pm 10.47^{\star \star * *}$ & $32.46 \pm 28.46^{\star \star * * \# \#}$ & 51.849 & $<0.001$ \\
\hline TBIL (mmol/L) & $10.35(7.50,13.48)$ & $12.00(9.50,15.48)^{\star}$ & $11.65(9.38,14.63)$ & $9.20(6.65,10.95)^{* * \#}$ & 25.074 & $<0.001$ \\
\hline $\mathrm{UA}(\mu \mathrm{mol} / \mathrm{L})$ & $290.38 \pm 84.65$ & $294.62 \pm 84.24$ & $293.80 \pm 98.07$ & $338.10 \pm 91.55^{\star * \#}$ & 3.441 & 0.017 \\
\hline $\mathrm{TNF}-\alpha / \operatorname{Ucr}(\mathrm{ng} / \mathrm{g})$ & $6.49 \pm 2.78$ & $8.09 \pm 4.36$ & $17.24 \pm 11.02 \star \star * *$ & $15.34 \pm 8.25^{\star \star * *}$ & 40.305 & $<0.001$ \\
\hline IL-18 /Ucr (ng/g) & $27.62(18.13,39.60)$ & $32.24(20.91,51.34)^{\star}$ & $\begin{array}{l}72.49(39.56 \\
113.18)^{\star \star * *}\end{array}$ & $93.28(40.71,141.29)^{\star \star * * \# \#}$ & 89.449 & $<0.001$ \\
\hline NLR & $1.39(1.10,2.17)$ & $1.53(1.16,2.09)$ & $1.92(1.33,2.83)^{\star *}$ & $2.22(1.71,3.89)^{\star \star * * \#}$ & 28.380 & $<0.001$ \\
\hline
\end{tabular}

3 BMI, body mass index; FBG, fasting blood glucose; Scr, serum creatinine; eGFR, estimated glomerular filtration

4 rate; TC, total cholesterol; TG, triglyceride; LDL, low-density lipoprotein; HbA1c, hemoglobin A1c; Tf, transferrin;

5 IgG, immunoglobulin G; PCX, podocalyxin; NGAL, neutrophil gelatinase-associated lipocalin; CysC, cystatin C;

6 NAG, N-acetyl-beta-glucosaminidase; alMG, alpha-1-microglobulin; 8-OHdG, 8-hydroxy-deoxyguanosine; TBIL,

7 total bilirubin; UA, uric acid; TNF- $\alpha$, tumor necrosis factor alpha; IL-18, interleukin-18; NLR, neutrophil to

8 lymphocyte ratio; Ucr, urinary creatinine. ${ }^{\star} \mathrm{P}<0.05$, ${ }^{\star \star} \mathrm{P}<0.001$ vs. healthy controls; ${ }^{*} \mathrm{P}<0.05,{ }^{* *} \mathrm{P}<0.001$ vs.

9 normoalbuminuria group; ${ }^{\#} \mathrm{P}<0.05,{ }^{\#} \mathrm{P}<0.001$ vs. microalbuminuria group. 
Table 2 (on next page)

Correlation analysis between the biomarkers (Tf, IgG, NGAL, and TNF- $\alpha$ ) and UACR in patients with type 2 diabetes. 
1 Table 2. Correlation analysis between the biomarkers (Tf, IgG, NGAL, and TNF- $\alpha$ ) and UACR 2 in patients with type 2 diabetes.

\begin{tabular}{|c|c|c|c|c|c|}
\hline & $\begin{array}{l}\text { Partial regression } \\
\text { coefficient } \\
\text { (B) }\end{array}$ & $\begin{array}{c}\text { Standard error } \\
\text { (SE) }\end{array}$ & $\begin{array}{c}\text { Standard partial } \\
\text { regression } \\
\text { coefficient }(\beta)\end{array}$ & $\mathrm{t}$ & $p$-value \\
\hline \multicolumn{6}{|l|}{$\mathrm{Tf}$} \\
\hline unadjusted & 14.351 & 0.871 & 0.870 & 16.483 & $<0.001$ \\
\hline adjusted & 14.006 & 0.898 & 0.849 & 15.589 & $<0.001$ \\
\hline \multicolumn{6}{|l|}{ IgG } \\
\hline unadjusted & 1.113 & 0.211 & 0.225 & 5.262 & $<0.001$ \\
\hline adjusted & 1.070 & 0.220 & 0.216 & 4.860 & $<0.001$ \\
\hline \multicolumn{6}{|l|}{ NGAL } \\
\hline unadjusted & 0.126 & 0.032 & 0.158 & 3.970 & $<0.001$ \\
\hline adjusted & 0.127 & 0.032 & 0.158 & 3.943 & $<0.001$ \\
\hline \multicolumn{6}{|l|}{ TNF- $\alpha$} \\
\hline unadjusted & -2.090 & 0.295 & -0.356 & -7.086 & $<0.001$ \\
\hline adjusted & -1.939 & 0.308 & -0.330 & -6.294 & $<0.001$ \\
\hline
\end{tabular}

3 Tf, transferrin; IgG, immunoglobulin G; NGAL, neutrophil gelatinase-associated lipocalin; TNF- $\alpha$, tumor necrosis 4 factor alpha.

5 


\section{Table 3(on next page)}

Assessment of Tf, IgG, NGAL, and TNF- $\alpha$ in the diagnosis of early-stage diabetic nephropathy in patients with type 2 diabetes. 
1 Table 3. Assessment of Tf, IgG, NGAL, and TNF- $\alpha$ in the diagnosis of early-stage diabetic 2 nephropathy in patients with type 2 diabetes.

\begin{tabular}{lcccccc}
\hline Parameters & AUC & $95 \%$ CI & Cut-off & $\begin{array}{c}\text { Sensitivity } \\
(\%)\end{array}$ & $\begin{array}{c}\text { Specificity } \\
(\%)\end{array}$ & $p$-value \\
\hline Tf & 0.861 & $0.810-0.902$ & 3.49 & 70.21 & 87.41 & $<0.001$ \\
IgG & 0.894 & $0.848-0.930$ & 8.56 & 75.53 & 92.31 & $<0.001$ \\
NGAL & 0.875 & $0.826-0.915$ & 30.03 & 84.04 & 74.13 & $<0.001$ \\
TNF- $\alpha$ & 0.763 & $0.704-0.816$ & 10.46 & 65.96 & 76.22 & $<0.001$ \\
Combination & 0.922 & $0.880-0.953$ & & 81.91 & 89.51 & $<0.001$ \\
UACR & 1.000 & 1.000 & 3.10 & 100.00 & 100.00 & $<0.001$ \\
\hline
\end{tabular}

3 Tf, transferrin; IgG, immunoglobulin G; NGAL, neutrophil gelatinase-associated lipocalin; TNF- $\alpha$, tumor necrosis 4 factor alpha; UACR, urine albumin to creatinine ratios. 
Figure 1

ROC curves for the diagnosis of early-stage diabetic nephropathy using urinary Tf, IgG,NGAL, and TNF- $\alpha$.

The abscissa represented specificity, the ordinate represented sensitivity, and the area under the curve represented the diagnostic value of urinary Tf, IgG,NGAL, and TNF- $\alpha$ in the earlystage diabetic nephropathy.

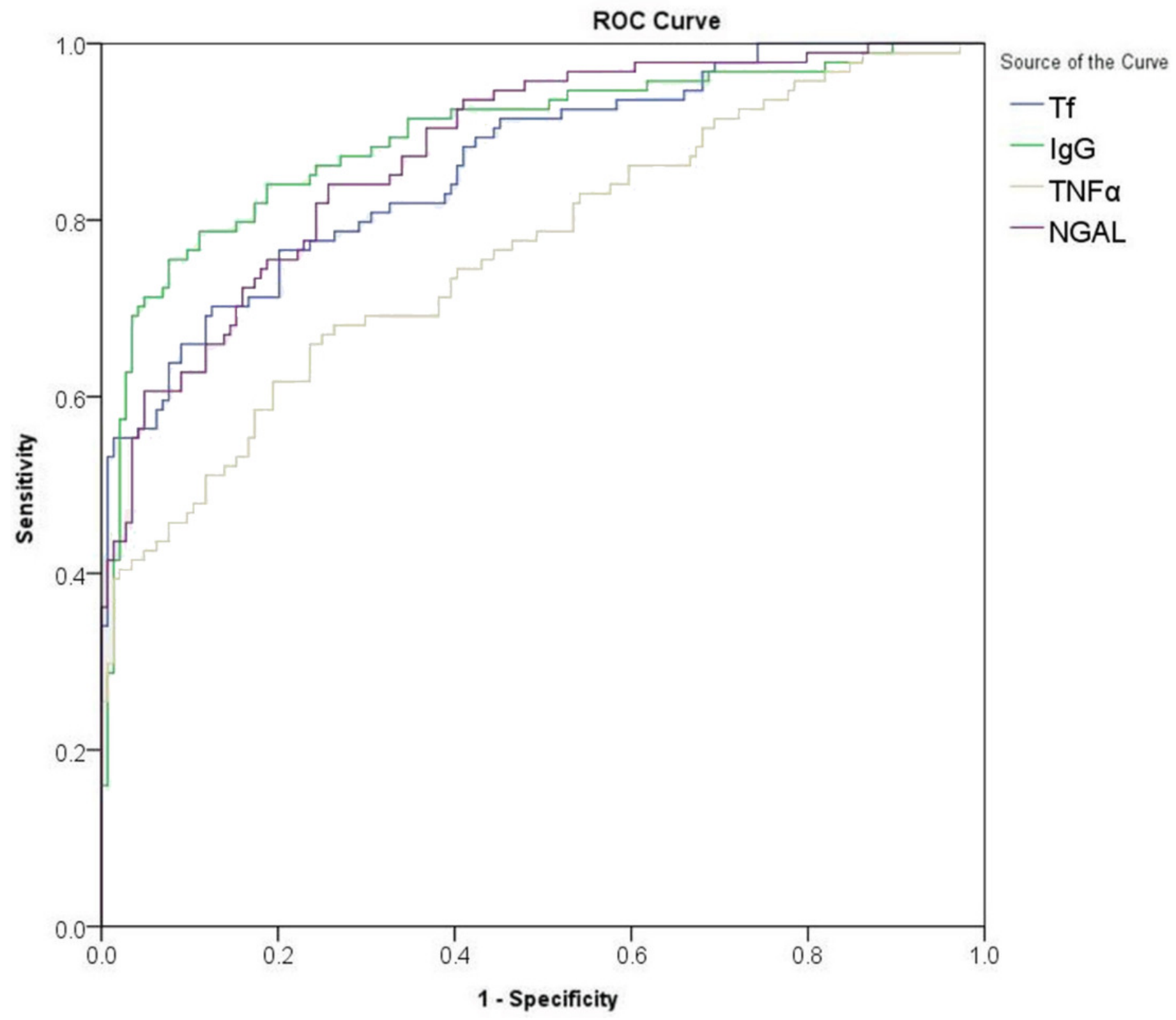


Figure 2

ROC curves for the diagnosis of early-stage diabetic nephropathy using the combination of urinary Tf, IgG,TNF- $\alpha$, and NGAL.

The abscissa represented specificity, the ordinate represented sensitivity, and the area under the curve represented the diagnostic value of the combination of urinary Tf, IgG,NGAL, and TNF- $\alpha$ in the early-stage diabetic nephropathy. 
ROC Curve

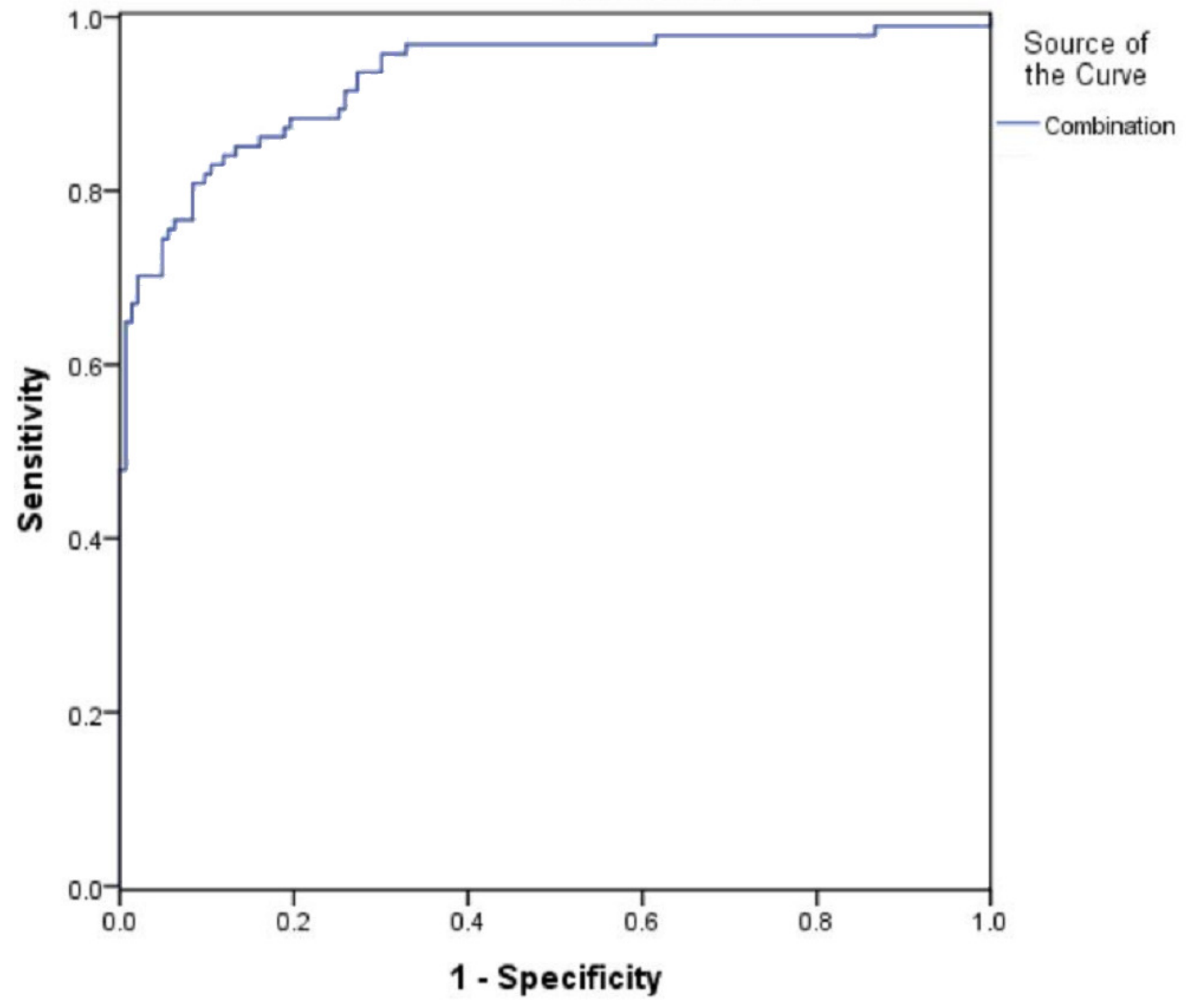

